\title{
Expression of protein disulfide isomerase A3 and its clinicopathological association in gastric cancer
}

\author{
TOMOHIRO SHIMODA ${ }^{1,2}$, RYUICHI WADA ${ }^{1,3}$, SHOKO KURE $^{1,3}$, KOUSUKE ISHINO $^{1}$, MITSUHIRO KUDO $^{1}$, \\ RYUJI OHASHI $^{4}$, ITSUO FUJITA ${ }^{2}$, EIJI UCHIDA ${ }^{2}$, HIROSHI YOSHIDA ${ }^{2}$ and ZENYA NAITO $^{1,3}$
}

\begin{abstract}
Departments of ${ }^{1}$ Integrated Diagnostic Pathology and ${ }^{2}$ Gastrointestinal and Hepato-Biliary-Pancreatic Surgery, Nippon Medical School, Tokyo 113-8602; ${ }^{3}$ Diagnostic Pathology, Nippon Medical School Hospital, Tokyo 113-8602; ${ }^{4}$ Department of Diagnostic Pathology, Nippon Medical School Musashi Kosugi Hospital, Kawasaki, Kanagawa 211-8533, Japan
\end{abstract}

Received September 24, 2018; Accepted January 24, 2019

DOI: $10.3892 /$ or.2019.6999

\begin{abstract}
Protein disulfide isomerase A3 (PDIA3) is a chaperone protein that supports the folding and processing of synthesized proteins. Its expression is associated with the prognosis of laryngeal cancer, hepatocellular carcinoma, diffuse glioma and uterine cervical cancer. In the present study, the expression levels of PDIA3 and its clinicopathological association were examined in 52 cases of gastric cancer (GC). The expression of PDIA3 was examined by immunohistochemistry and scored using a semi-quantitative method. According to the score, GC samples were classified into PDIA3-High and PDIA3-Low GC. PDIA3-High GC samples were predominantly of the intestinal type. Multivariate survival analysis indicated that PDIA3 expression and cancer stage were independent factors. The overall survival of PDIA3-High GC cases was significantly favorable compared with that of PDIA3-Low GC cases, and this was more evident in cases at an advanced stage. In GC cell cultures, the PDIA3 and major histocompatibility complex (MHC) class I proteins were expressed in three out of the four assessed cell lines according to western blot analysis. Notably, the expression of MHC class I was increased by the stimulation of interferon $\gamma$. Co-immunoprecipitation assays suggested the formation of a PDIA3 and MHC class I complex. The findings suggested that PDIA3 may be involved in the immune response of carcinoma cells. The improved
\end{abstract}

Correspondence to: Dr Ryuichi Wada, Department of Integrated Diagnostic Pathology, Nippon Medical School, 1-1-5 Sendagi, Bunkyo-ku, Tokyo 113-8602, Japan

E-mail:w-ryuichi@nms.ac.jp

Abbreviations: GC, gastric cancer; HLA, human leukocyte antigen; IFN $\gamma$, interferon $\gamma$; IHC, immunohistochemistry; IM, intestinal metaplasia; MHC, major histocompatibility complex; PBS, phosphate-buffered saline; PDIA3, protein disulfide isomerase A3; TBS-T, Tris-buffered saline/Tween-20

Key words: gastric cancer, protein disulfide isomerase A3, major histocompatibility complex class I, prognosis, stage, interferon $\gamma$ prognosis in PDIA3-High GC may be accounted for, in part, by sufficient antigen processing and expression of $\mathrm{MHC}$ class I, which can be mediated by PDIA3. It was suggested that PDIA3 serves an important role in the pathobiology of $\mathrm{GC}$, and that PDIA3 is a useful marker for the prediction of prognosis.

\section{Introduction}

Gastric cancer (GC) is the third major cause of cancer-associated fatality in Japan (1). The prognosis has improved because of early diagnosis and surgical treatment, followed by chemotherapy and molecular targeting therapy using anti-human epidermal growth factor receptor 2 antibody and anti-programmed death-ligand 1 antibody (2). However, the prognosis of GC at an advanced stage is still unfavorable (3). New strategies for the diagnosis and treatment of GC are therefore required.

Protein disulfide isomerase A3 (PDIA3), which is also known as GRP58/ERp57, is a chaperone protein that supports the folding and processing of protein synthesized in the endoplasmic reticulum (4). PDIA3 is involved in multiple biological functions, including stabilization of receptors, antigen processing and presentation, and degradation of proteins (5). It has also been indicated that PDIA3 is involved in the proliferation and cell death in human carcinomas (6). High expression levels of PDIA3 are associated with poor prognosis of laryngeal cancer, hepatocellular carcinoma and diffuse glioma (7-9). However, high expression levels of PDIA3 are also associated with a favorable prognosis of uterine cervical cancer (10). Regarding GC, it has been reported that high expression of PDIA3 is associated with favorable prognosis in GC at early stage; however, an association has not been identified in GC at the advanced stage (11). The association of PDIA3 with prognosis of GC in Japanese cases is not known. Furthermore, the mechanism of favorable prognosis in $\mathrm{GC}$ with high expression of PDIA3 remains undetermined.

In the present study, the association of PDIA3 expression with clinicopathological features, including prognosis, was investigated in Japanese cases of GC. A possible mechanism of how PDIA3 affects the prognosis of GC was also suggested. 


\section{Materials and methods}

Cases of GC. Cases of GC were retrieved from the archives of the pathology records of Nippon Medical School Hospital (Tokyo, Japan) between January 2006 and December 2008. A total of 52 cases were used for the study. The cases were randomly selected from the cases of GC. The patients did not receive chemotherapy or radiation prior to the surgery. Clinical information and the time of death following surgery were retrieved from the clinical records. The study was conducted according to the declaration of Helsinki and the Japanese Society of Pathology. The study was approved by the Ethics Committee of the Nippon Medical School Hospital (Tokyo, Japan; no. 29-06-764). Informed consent was obtained from all patients.

Histological examination of GC. The histology of the pathological specimens of GC was reviewed by three individuals. Resected stomachs were fixed in $10 \%$ formalin at room temperature for $24 \mathrm{~h}$ and processed to be embedded in paraffin. Three $\mu \mathrm{m}$-thick sections of the entire area of the carcinoma were stained with hematoxylin and eosin (stained for $5 \mathrm{~min}$ with hematoxylin and 3 min with eosin at room temperature) and observed using microscope (ECLIPSE 50i; Nikon Corp., Tokyo, Japan). The histological subtype was determined as the predominant histology of the carcinoma tissue according to the classification indicated by Laurén (12). The depth of the invasion and lymph node metastasis were also evaluated (13). The pathological stage was classified according to the classification of the Union for International Cancer Control (UICC) (14). Helicobacter pylori infection state was evaluated with $3 \mu \mathrm{m}$-thick sections stained with hematoxylin and eosin and viewed using a microscope (ECLIPSE 50i; Nikon Corp.) at high magnification $(\mathrm{x} 1,000)$. The infection state was also considered positive if the serum was positive for anti-Helicobacter pylori antibody.

Immunohistochemistry and semi-quantitative evaluation. Immunohistochemistry was conducted using a polymer-based two-step method. Briefly, 3- $\mu$ m-thick paraffin sections were deparaffinized and hydrated in phosphate-buffered saline (PBS). Sections were pretreated in Tris- $\mathrm{HCl}(\mathrm{pH} \mathrm{9.0)}$ for PDIA 3 and citrate buffer $(\mathrm{pH} 6.0)$ for $\mathrm{Ki}-67$ at $121^{\circ} \mathrm{C}$ for 15 min. Endogenous peroxidase was blocked in $3 \% \mathrm{H}_{2} \mathrm{O}_{2}$ in methanol at room temperature for $30 \mathrm{~min}$. The sections were subsequently incubated with mouse anti-PDIA3 antibody (cat. no. ab13506; dilution, 1:2,000; Abcam K.K., Tokyo, Japan) and mouse anti-Ki-67 antibody (cat. no. MIB1; dilution, 1:100; Agilent Technologies Japan, Ltd., Tokyo, Japan) overnight at $4^{\circ} \mathrm{C}$. Following this, the sections were incubated with Histofine Simple Stain MAX-PO (M) (Nichirei Bioscience Inc., Tokyo, Japan) at room temperature for $30 \mathrm{~min}$. The peroxidase activity was visualized using diaminobenzidine at room temperature for $2 \mathrm{~min}$.

The intensity and proportion of stained carcinoma cells were scored using a semi-quantitative method (15). The immunostained sections were observed using a microscope (ECLIPSE 50i; Nikon Corp.) at a magnification of x400. Cytoplasmic staining was considered to indicate a positive reaction. The intensity score was divided into four grades as follows: no staining, score of 0 ; moderate staining similar to that of the cells in the glands with intestinal metaplasia (IM), score of 2; clear but weaker staining than that of the metaplastic cells, score of 1 ; and stronger staining than that of these cells, score of 3. Furthermore, the proportion of positive cells of each intensity score was evaluated as a percentage in $10 \%$ increments. The total score was calculated using the following formula: $1 \mathrm{x}$ (proportion of score 1 cells) $+2 \mathrm{x}$ (proportion of score 2 cells) $+3 \times$ (proportion of score 3 cells). Two individuals evaluated the intensity score and proportion of the positive cells in a blind manner. When the values were discrepant, the two individuals discussed the results and the most appropriate value was determined.

The Ki-67 labeling index was calculated as the percentage of Ki-67-positive cells in $~ 1,000$ tumor cells in the areas of the highest nuclear labeling under $\mathrm{x} 400$ magnification using the eCount image analysis software version 4.7 (e-Path Co., Ltd., Fujisawa, Kanagawa, Japan).

Terminal deoxynucleotidyl transferase dUTP nick-end labeling (TUNEL) assay. Apoptotic cell death was determined by TUNEL assay using the Apoptag Peroxidase In Situ Apoptosis Detection Kit (EMD Millipore, Temecula, CA, USA). Briefly, deparaffinized 3- $\mu \mathrm{m}$-thick paraffin section was digested with $20 \mu \mathrm{g} / \mathrm{ml}$ proteinase K (cat. no. S3004; Agilent Technologies Japan, Ltd.) at $37^{\circ} \mathrm{C}$ for $30 \mathrm{~min}$. The labeling was performed in the mixture of terminal deoxyribonucleotide transferase and digoxygenin-dUTP at $37^{\circ} \mathrm{C}$ for $1 \mathrm{~h}$. Incorporated digoxygenin-dUTP was detected by peroxidase-labeled anti-digoxygenin antibody, which was included in the kit. The peroxidase activity was visualized using diaminobenzidine at room temperature for $2 \mathrm{~min}$. Nuclear staining was considered positive. The TUNEL index was calculated as the percentage of TUNEL-positive cells in 1,000 counted carcinoma cells in the areas of highest nuclear labeling under $\mathrm{x} 400$ magnification using the eCount image analysis software version 4.7 (e-Path Co., Ltd.).

Extraction of total RNA and reverse transcription-quantitative polymerase chain reaction ( $R T-q P C R)$. Total RNA was extracted from paraffin sections of GCs. The sections were deparaffinized and hydrated. Following this, carcinoma tissues were dissected and collected into $1.5-\mathrm{ml}$ tubes. For the standardization, normal mucosa with IM were used. Total RNA was extracted using the RNeasy FFPE Kit (Qiagen, K.K., Tokyo, Japan) following the protocol recommended by the manufacturer, and the concentration of total RNA was quantified. cDNA was synthesized by random primer method using SuperScript VILO cDNA Synthesis Kit (Thermo Fisher Scientific, K.K., Tokyo, Japan). The qPCR was performed with a $20-\mu 1$ mixture of $1 \mathrm{X}$ TaqMan Master Mix (Thermo Fisher Scientific, K.K.), the primers and probes of PDIA3 (Hs04194196) or 18S rRNA (Hs03928990) (both from Thermo Fisher Scientific, K.K.) and cDNA synthesized from $20 \mathrm{ng}$ of total RNA. The sequences of primers and probes used for the TaqMan assay are not published by the company. The reaction program was initiated at $95^{\circ} \mathrm{C}$ for $20 \mathrm{sec}$, followed by 40 cycles of $95^{\circ} \mathrm{C}$ for $1 \mathrm{sec}$ and $60^{\circ} \mathrm{C}$ for $20 \mathrm{sec}$. The changes in fluorescence were monitored using a StepOne Plus Real-Time PCR System (Thermo Fisher Scientific, K.K.). Quantification cycles 
(Cq) of PDIA3 and 18S rRNA were determined as the cycle where the linear increase in fluorescence reached the threshold level. For standardization, $\Delta \mathrm{Cq}$ was calculated by subtracting $\mathrm{Cq}_{18 \mathrm{~s} \text { rRNA }}$ from $\mathrm{Cq}_{\text {PDIA3 }}$. Then, $\Delta \Delta \mathrm{Cq}$ was calculated by the subtraction of $\Delta \mathrm{Cq}$ of IM from $\Delta \mathrm{Cq}$ of GC. The relative expression levels were calculated using the $2^{-\Delta \Delta \mathrm{Cq}}$ method (16). The expression levels of PDIA3 mRNA in GC were calculated as fold expression relative to IM.

Cell culture. The experiment was performed with four cell lines of GC: NS-8, MKN-7, NUGC-4 and KATO-III (Cell Resource Center for Biomedical Research Institute of Development, Aging and Cancer, Tohoku University, Sendai, Japan). The cells were cultured in Dulbecco's Modified Eagle's medium (DMEM; Thermo Fisher Scientific, K.K.) supplemented with $10 \%$ fetal bovine serum (Nichirei Bioscience Inc.). The cells were cultured in the medium with $100 \mathrm{ng} / \mathrm{ml}$ interferon $\gamma$ (IFN $\gamma$; cat. no. 80385; Cell Signaling Technology Japan, K.K., Tokyo, Japan) for 48 h. Following a wash step with PBS, the cells were lysed in a $0.5 \% \mathrm{SDS} / 50 \mathrm{mM}$ Tris- $\mathrm{HCl}$ (pH 7.6) buffer and sonicated for $10 \mathrm{~min}$. The protein concentration was quantified using a Pierce $660 \mathrm{~nm}$ Protein Assay Reagent (Thermo Fisher Scientific, K.K.) and used for western blot analysis.

Co-immunoprecipitation analysis. KATO-III cells were cultured in DMEM supplemented with $100 \mathrm{ng} / \mathrm{ml} \mathrm{IFN} \gamma$ for $48 \mathrm{~h}$, and the cells were collected into $15-\mathrm{ml}$ tubes with a cell scraper. The cells were washed with PBS and centrifuged at $1,500 \times \mathrm{g}$ at $4^{\circ} \mathrm{C}$ for $5 \mathrm{~min}$. Then, the cells were lysed with immunoprecipitation (IP) lysis/wash buffer (Thermo Fisher Scientific, K.K.) with protease inhibitor cocktail (cat. no. P8340; dilution, 1:100; Sigma-Aldrich Japan K.K., Tokyo, Japan), and incubated on ice for $10 \mathrm{~min}$. The solution was centrifuged at $12,000 \mathrm{x}$ g at $4^{\circ} \mathrm{C}$ for $5 \mathrm{~min}$. The supernatant was transferred to a new tube, and the protein concentration was quantified using a Pierce $660 \mathrm{~nm}$ Protein Assay Reagent (Thermo Fisher Scientific, K.K.). Protein from each cell line $(1 \mathrm{mg})$ was incubated in $800 \mu \mathrm{l}$ IP lysis/wash buffer mixed with Protein A/G PLUS-Agarose (Santa Cruz Biotechnology, Inc., Santa Cruz, CA, USA) and $2 \mu \mathrm{g}$ of anti-PDIA3 antibody (cat. no. ab13506; dilution, 1:400; Abcam K.K.), anti-HLA class I-A, B, C antibody (cat. no. AB-46-H; dilution, 1:400; Hokudo System Co. Ltd., Sapporo, Japan) and isotype normal mouse IgG (cat. no. SC2025; dilution, 1:320; Santa Cruz Biotechnology, Inc.) at $4^{\circ} \mathrm{C}$ overnight. The mixture was subsequently applied to Sigma Prep Spin Columns with Break-Away Tips (Sigma-Aldrich Japan K.K.), and the column was washed with a buffer of $0.01 \mathrm{M}$ Tris- $\mathrm{HCl}(\mathrm{pH} 7.6) / 150 \mathrm{mM} \mathrm{NaCl} / 0.05 \%$ Tween-20 (TBS-T) three times. The proteins were eluted with $30 \mu 1$ of Laemmli Sample Buffer (Bio-Rad Laboratories, Inc., Tokyo, Japan) with 3-mercaptethanol and used for western blot analysis.

Western blot analysis. The protein samples were electrophoresed in a 5-20\% gradient gel (e-PAGEL; cat. no. E-T520L; ATTO Corporation, Tokyo, Japan). For the analysis of protein expression in cultured cells, $10 \mu \mathrm{g}$ of cell lysates were mixed with Laemmli Sample Buffer (Bio-Rad Laboratories, Inc.) and loaded to the wells. For the analysis of immunoprecipitated proteins, $20 \mu \mathrm{l}$ of eluted solutions were loaded to the wells. The electrophoresed proteins were blotted onto a polyvinylidene difluoride membrane. Following the blocking of the membrane with 5\% skim milk in TBS-T at room temperature for $1 \mathrm{~h}$, the membrane was incubated with monoclonal antibodies at $4^{\circ} \mathrm{C}$ overnight. The antibodies used in the analysis were anti-PDIA3 (cat. no. ab13506; dilution 1:2,000; Abcam K.K.), anti-HLA class I-A, B, C antibody (cat. no. AB-46-H; dilution, 1:500; Hokudo System Co. Ltd.) and anti- $\beta$-actin antibody (cat. no. A5316; dilution, 1:10,000; Sigma-Aldrich Japan K.K.). The membrane was subsequently incubated with horse radish peroxidase-labeled anti-mouse $\mathrm{IgG}$ antibody (TrueBlot; cat. no. 18-8817-30; dilution, 1:10,000; Rockland Immunochemicals Inc., Pottstown, PA, USA) at room temperature for $1 \mathrm{~h}$. The peroxidase activity was detected as chemiluminescence using SuperSignal West Dura Extended Duration Substrate (Thermo Fisher Scientific, K.K.). The positive bands were quantified using Quantity One Software version 4.6.2 (Bio-Rad Laboratories, Inc.).

Statistical analysis. Data were indicated as the mean \pm standard deviation. The data of two groups were compared using the Mann-Whitney U test. Clinicopathological parameters were analyzed using the $\chi^{2}$ and Fisher's exact tests. The Cox proportional hazards model was used to identify independent factors, which had a significant influence on the survival. Overall survival was analyzed by the Kaplan-Meier's method and the $\log$ rank test. $\mathrm{P}<0.05$ was considered to indicate a statistically significant difference. All statistical analyses were performed using SPSS (version 23; IBM Corp., Armonk, NY, USA).

\section{Results}

Cases of GC. A total of 52 cases of GC were retrieved from the archives of pathology records. The ages of the cases used in the present study ranged between 45 and 81 years old. Thirty-five men and 17 women represented the cases. The pathological stages ranged from I to IV. The follow-up duration ranged between 6 and 92 months (mean, 59 months). Representative histology images of diffuse and intestinal type GC were indicated (Fig. 1A-C). Twenty-three cases were classified into the intestinal type and 29 cases were considered the diffuse type. Ki-67 labeling index ranged from 2.1 to $97.3 \%$ (Fig. 1D). Apoptotic cell death, which was evaluated by TUNEL, ranged from 1.3 to $37.1 \%$ (Fig. 1E). Infection of Helicobacter pylori was noted in 45 (88\%) cases (Fig. 1F). Lymph node metastasis (Fig. 1G) and distant metastasis were identified in $29 / 52(56 \%)$ cases and 10/52 (19\%) cases, respectively.

PDIA3 expression in GC samples. Glandular cells of the IM exhibited a positive reaction for PDIA3 (Fig. 2). The positive staining was observed in the cytoplasm of the carcinoma cells; however, their staining intensity varied largely (Fig. 2). Well-differentiated carcinomas tended to exhibit an intense positive reaction, whereas poorly differentiated adenocarcinomas and signet ring cells exhibited only a weak or faint positive reaction. The score of PDIA3 expression ranged between 0 and 300, and the median was 95. Cases with the total score $\geq 95$ were classified as PDIA3-High and those with a total score $<95$ were classified as PDIA3-Low. 

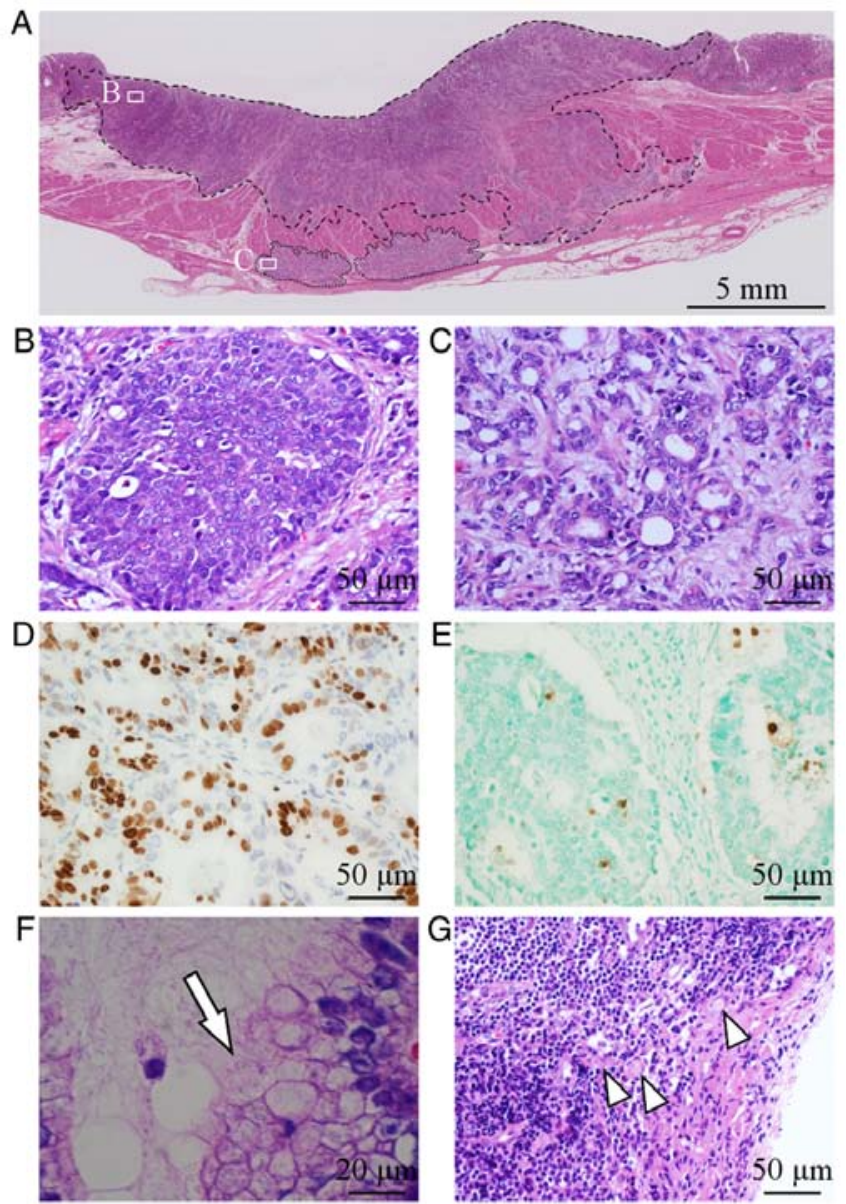

Figure 1. Representative histology of gastric cancer. (A) Representative image of gastric cancer (magnification, x0.3). High magnification of predominant histology of (B) diffuse type and (C) focal area of intestinal type (magnification, $\mathrm{x} 400$ ). Positive nuclear staining of (D) Ki-67 and (E) terminal deoxynucleotidyl transferase dUTP nick-end labeling method (magnification, $\mathrm{x} 400$ ). (F) Infection of Helicobacter pylori (arrow) in the gastric mucosa (magnification, $\mathrm{x} 1,000)$. (G) Lymph node metastasis of signet ring cells (arrow heads) (magnification, $\mathrm{x} 400$ ).

Expression of PDIA3 mRNA in GC samples. The expression levels of PDIA3 were further verified by RT-qPCR. The expression levels of mRNA in PDIA3-High GC samples were significantly elevated compared with PDIA3-Low GC samples (Fig. 3).

Clinicopathological features of GC. The clinicopathological features are summarized in Table I. The intestinal type was the predominant histopathological subtype in PDIA3-High GC, whereas the diffuse type was predominant in PDIA3-Low GC. There was no significant difference in the frequency of Ki-67 labeling and TUNEL staining between PDIA3-High and PDIA3-Low GC (Fig. 4). There was also no significant difference in other assessed clinicopathological features between PDIA3-High and PDIA3-Low GC.

Survival analysis of GC. The overall survival of PDIA3-High and PDIA3-Low GC cases was analyzed using univariate and multivariate analyses. With univariate analysis, the hazard ratio of the histological subtype, PDIA3 expression and UICC stage were significantly increased (Table II). In multivariate
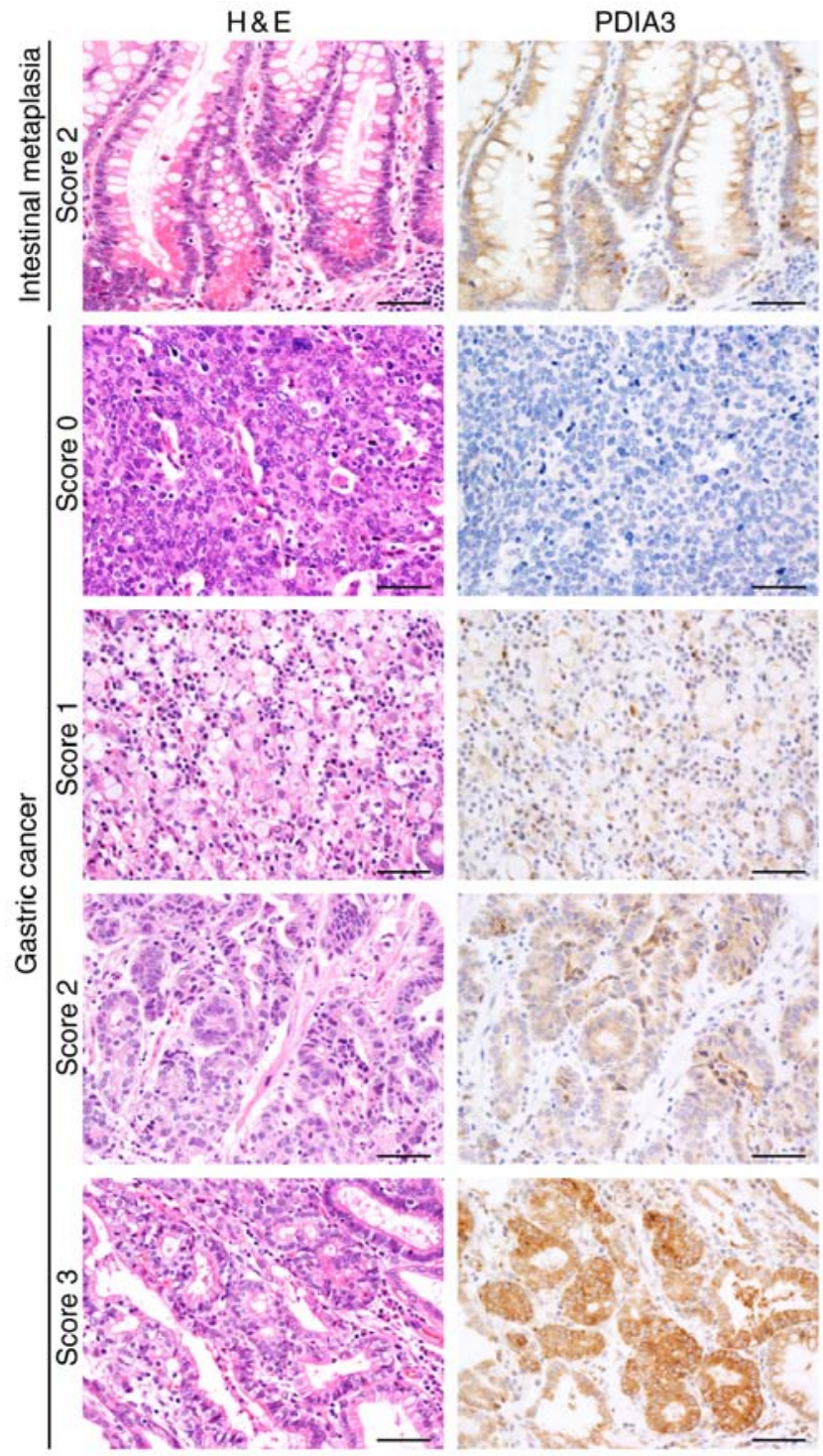

Figure 2. Immunohistochemistry of PDIA3. Representative images of hematoxylin and eosin staining and expression of PDIA3 in gastric cancer and normal gastric mucosa. The cytoplasmic reaction in the cells of intestinal metaplasia was given a score of 2 . The expression of PDIA3 in diffuse type GC appeared low, whereas the expression in intestinal type GC appeared high. All images are $\mathrm{x} 400$ magnification. Scale bars are $50 \mu \mathrm{m}$. PDIA3, Protein disulfide isomerase A3; GC, gastric cancer.

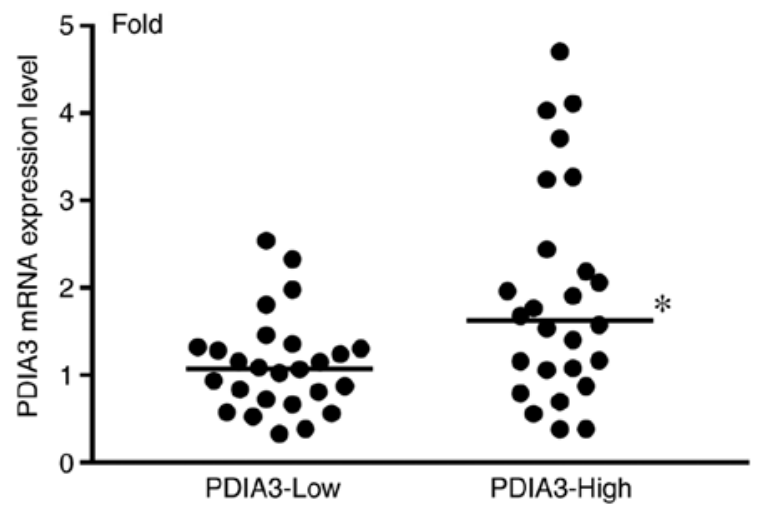

Figure 3. PDIA 3 mRNA expression levels in gastric cancer. The mRNA levels of PDIA3 were increased in PDIA3-High gastric cancer cases compared with that in PDIA3-Low gastric cancer cases. ${ }^{*} \mathrm{P}<0.05$ vs. PDIA3-Low gastric cancer. PDIA3, protein disulfide isomerase A3. 
Table I. Association of PDIA3 with clinicopathological features of 52 cases of gastric cancer.

\begin{tabular}{|c|c|c|c|c|}
\hline \multirow[b]{2}{*}{ Factors } & \multirow[b]{2}{*}{ Cases } & \multicolumn{2}{|c|}{$\begin{array}{c}\text { PDIA3 } \\
\text { expression }\end{array}$} & \multirow[b]{2}{*}{ P-value } \\
\hline & & High & Low & \\
\hline \multicolumn{5}{|l|}{ Age, years } \\
\hline$\geq 65$ & 29 & 15 & 14 & \multirow[t]{2}{*}{0.780} \\
\hline$<65$ & 23 & 11 & 12 & \\
\hline \multicolumn{5}{|l|}{ Sex } \\
\hline Male & 35 & 18 & 17 & \multirow[t]{2}{*}{0.768} \\
\hline Female & 17 & 8 & 9 & \\
\hline \multicolumn{5}{|l|}{ Location } \\
\hline Upper & 9 & 4 & 5 & \multirow[t]{3}{*}{0.697} \\
\hline Middle & 22 & 10 & 12 & \\
\hline Lower & 21 & 12 & 9 & \\
\hline \multicolumn{5}{|c|}{ Histological subtype } \\
\hline Intestinal type & 23 & 17 & 6 & \multirow[t]{2}{*}{0.002} \\
\hline Diffuse type & 29 & 9 & 20 & \\
\hline \multicolumn{5}{|c|}{$\mathrm{Ki}-67$ labeling index } \\
\hline$\geq 55.6 \%$ & 26 & 15 & 11 & \multirow[t]{2}{*}{0.267} \\
\hline$<55.6 \%$ & 26 & 11 & 15 & \\
\hline \multicolumn{5}{|l|}{ TUNEL index } \\
\hline$\geq 6.1 \%$ & 26 & 15 & 11 & \multirow[t]{2}{*}{0.267} \\
\hline$<6.1 \%$ & 26 & 11 & 15 & \\
\hline \multicolumn{5}{|c|}{$\begin{array}{l}\text { Helicobacter pylori } \\
\text { infection }\end{array}$} \\
\hline Positive & 45 & 22 & 23 & \multirow[t]{2}{*}{0.500} \\
\hline Negative & 7 & 4 & 3 & \\
\hline \multicolumn{5}{|c|}{ Depth of tumor invasion } \\
\hline pT1 & 21 & 11 & 10 & \multirow[t]{4}{*}{0.748} \\
\hline pT2 & 3 & 1 & 2 & \\
\hline pT3 & 8 & 5 & 3 & \\
\hline pT4 & 20 & 9 & 11 & \\
\hline \multicolumn{5}{|c|}{ Lymph node metastasis } \\
\hline Positive & 29 & 16 & 13 & \multirow[t]{2}{*}{0.402} \\
\hline Negative & 23 & 10 & 13 & \\
\hline \multicolumn{5}{|c|}{ Distant metastasis } \\
\hline Positive & 10 & 3 & 7 & \multirow[t]{2}{*}{0.159} \\
\hline Negative & 42 & 23 & 19 & \\
\hline \multicolumn{5}{|l|}{ UICC stage } \\
\hline I & 21 & 10 & 11 & \multirow[t]{4}{*}{0.402} \\
\hline II & 7 & 4 & 3 & \\
\hline III & 14 & 9 & 5 & \\
\hline IV & 10 & 3 & 7 & \\
\hline
\end{tabular}

UICC, Union for International Cancer Control; TUNEL, terminal deoxynucleotidyl transferase dUTP nick-end labeling.

analysis, PDIA3 expression and UICC stage were determined as independent factors (Table III).
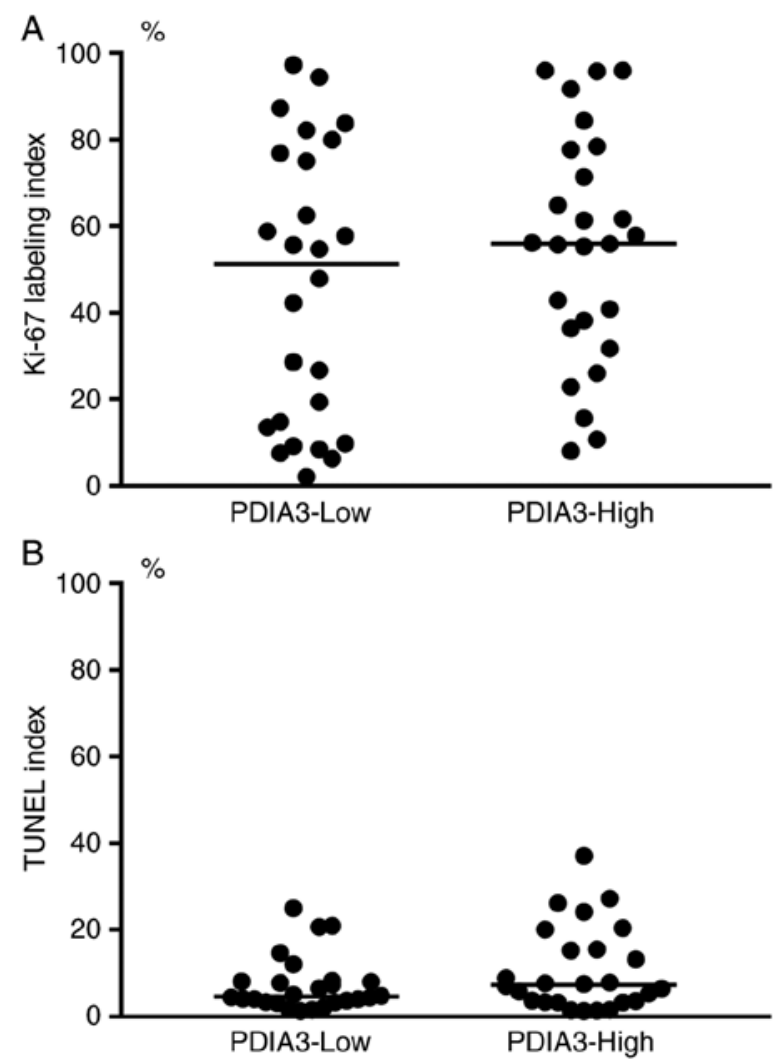

Figure 4. Ki-67 labeling index and TUNEL index in gastric cancer. (A) There was no significant difference in the $\mathrm{Ki}-67$ labeling index between PDIA3-High and PDIA3-Low gastric cancer cases. (B) TUNEL index was not significantly different between PDIA3-High and PDIA3-Low gastric cancer cases. TUNEL, terminal deoxynucleotidyl transferase dUTP nick-end labeling; PDIA3, protein disulfide isomerase A3.

The overall survival of PDIA3-High GC cases was significantly favorable compared with that in PDIA3-Low GC cases (Fig. 5A). Survival was analyzed in the early and advanced stages. In the early stage, survival tended to be worse in PDIA3-Low GC cases (Fig. 5B). In the advanced stage, the survival was significantly favorable in PDIA3-High GC cases. The 5-year survival was 75\% (Fig. 5C). Survival was significantly worse in PDIA3-Low GC cases, and 5-year survival of this subset was $27 \%$.

Expression of PDIA3 and MHC class I in GC culture cells. The expression levels of PDIA3 and MHC class I were examined in four cell lines, GC, NS-8, MKN-7, NUGC-4 and KATO-III (Fig. 6). The expression levels of these proteins were also examined in cells under the stimulation of IFN $\gamma$, which is known to induce the expression of MHC class I (17). PDIA3 was not expressed in NS-8. However, PDIA3 was expressed in the other three cell lines, and the expression level appeared to be lower in MKN-7 and NUGC-4 compared with KATO-III. The expression of MHC class I was identified in all cell lines except NS-8. Under the stimulation of IFN $\gamma$, the expression level of PDIA3 appeared to be increased in KATO-III; however, the upregulation was not evident in IFN $\gamma$-treated MKN-7 and NUGC-4. Notably, the expression of MHC class I was increased by 4.5-, 18.3-, and 3.5-fold in IFN $\gamma$-treated MKN-7, NUGC-4 and KATO-III, respectively (8.8 \pm 8.3 -fold on average). 
Table II. Univariate survival analysis of overall survival in 52 cases of gastric cancer.

\begin{tabular}{|c|c|c|c|}
\hline Factors & Hazard ratio & $95 \% \mathrm{CI}$ & P-value \\
\hline Age, years $(<65$ vs. $\geq 65)$ & 0.58 & $0.23-1.47$ & 0.248 \\
\hline Sex (male vs. female) & 0.80 & $0.29-2.24$ & 0.672 \\
\hline Location (upper vs. middle/lower) & 0.73 & $0.24-2.22$ & 0.578 \\
\hline Histological subtype (intestinal vs. diffuse) & 3.20 & $1.05-9.79$ & 0.042 \\
\hline Ki-67 labeling index $(<55.6$ vs. $\geq 55.6 \%)$ & 1.66 & $0.64-4.29$ & 0.295 \\
\hline TUNEL index $(<6.1$ vs. $\geq 6.1 \%)$ & 0.63 & $0.24-1.63$ & 0.340 \\
\hline PDIA3 (low vs. high) & 4.51 & $1.47-13.78$ & 0.008 \\
\hline Helicobacter pylori infection (negative vs. positive) & 0.62 & $0.18-2.15$ & 0.454 \\
\hline UICC stage (I vs. II/III/IV) & 8.01 & $1.83-35.07$ & 0.006 \\
\hline
\end{tabular}

CI, confidence interval; UICC, Union for International Cancer Control; TUNEL, terminal deoxynucleotidyl transferase dUTP nick-end labeling; PDIA3, protein disulfide isomerase A3.

Table III. Multivariate survival analysis of overall survival in 52 cases of gastric cancer.

\begin{tabular}{lccc}
\hline Factors & Hazard ratio & $95 \%$ CI & P-value \\
\hline $\begin{array}{l}\text { Histological subtype } \\
\text { (intestinal vs. diffuse) }\end{array}$ & 1.24 & $0.38-4.09$ & 0.721 \\
PDIA3 (low vs. high) & 5.56 & $1.67-18.46$ & 0.005 \\
Stage (I vs. II/III/IV) & 10.13 & $2.26-45.36$ & 0.002 \\
\hline
\end{tabular}

CI, confidence interval; UICC, Union for International Cancer Control.

Co-immunoprecipitation analysis. The formation of a PDIA3 and MHC class I complex was examined in the KATO-III cell line stimulated with IFN $\gamma$ (Fig. 7). Western blot analysis with anti-PDIA3 antibody revealed a clear positive signal in the sample immunoprecipitated with anti-PDIA3 antibody and a faint band in the sample immunoprecipitated with anti-MHC class I antibody. Western blot analysis with an anti-MHC class I antibody indicated a clear band in the sample immunoprecipitated with anti-MHC class I antibody and a faint band in the sample immunoprecipitated with anti-PDIA3 antibody. These results suggested that PDIA3 and MHC class I formed a complex in KATO-III cells.

\section{Discussion}

The present study demonstrated the expression of PDIA3 in GC and a favorable prognosis of PDIA3-High GC. The results are in line with a previous report that also presented a favorable prognosis of GC highly expressing PDIA3 (11). However, there is a slight difference in prognosis between the previous and present study. In the previous study, the prognosis was favorable in the cases at early stage but not in the cases at advanced stage. The present study demonstrated a significantly favorable prognosis in PDIA3-High GC of all stages. In the cases of stage I, the prognosis appeared to be improved in PDIA3-High $\mathrm{GC}$, although there was no statistical significance. In the cases
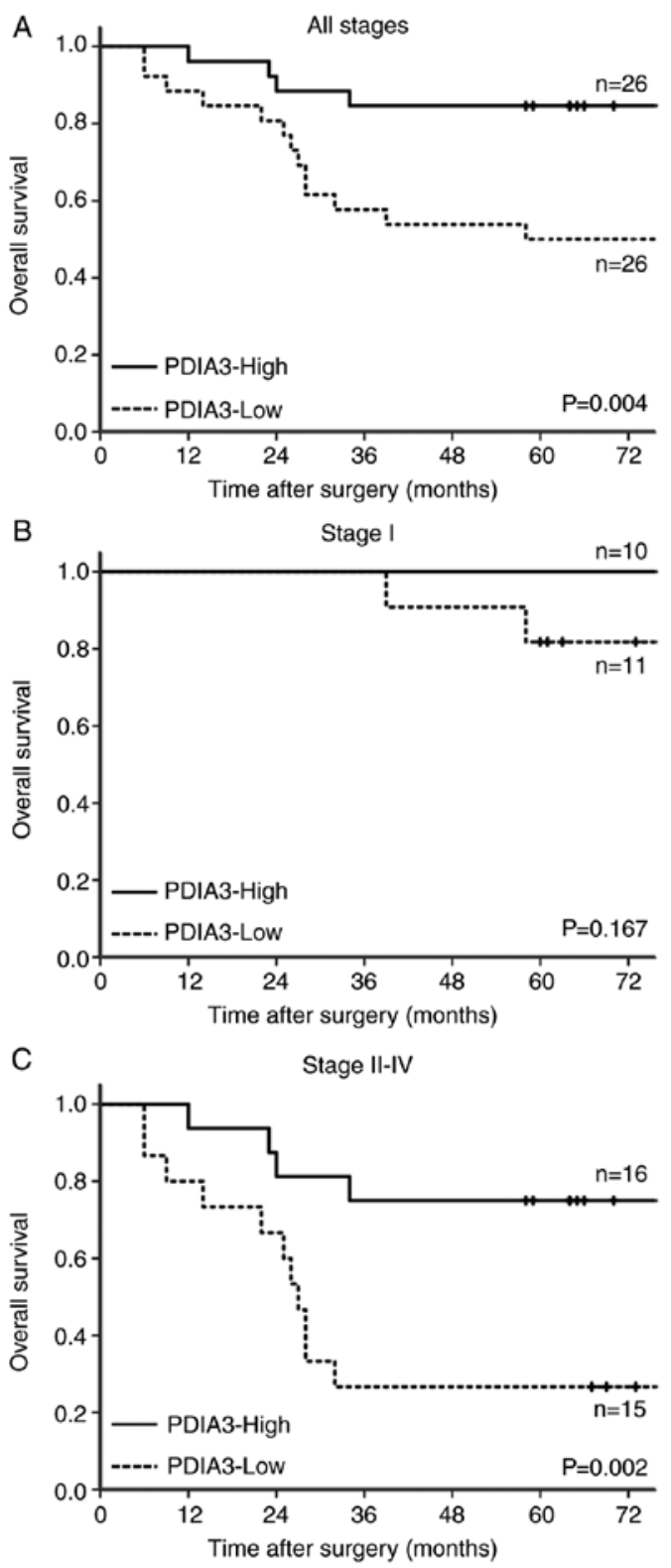

Figure 5. Overall survival of gastric cancer with PDIA3-High and PDIA3-Low gastric cancer cases. The overall survival of the cases of (A) all stages, (B) at stage I and (C) at stages II to IV. PDIA3, protein disulfide isomerase A3. 


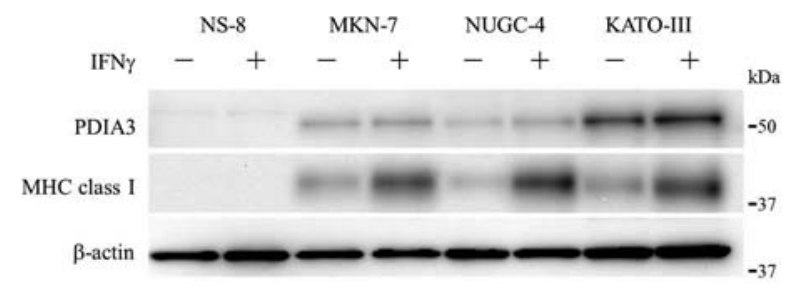

Figure 6. Western blot analysis of gastric cancer cell lines. PDIA3 and MHC class I proteins were expressed in three out of four cell lines. The expression level of PDIA3 in MKN-7 and NUGC-4 was weak, whereas the expression level was elevated in KATO-III. Expression was not observed in NS-8. Under the stimulation of interferon $\gamma$, the expression level of PDIA3 in KATO-III appeared to increase. The expression of MHC class I was noted in the MKN-7, NUGC-4 and KATO-III cell lines. Its expression was increased in these cell lines, following the stimulation of interferon $\gamma$, and the mean expression level was increased by 8.8 -fold. PDIA3, protein disulfide isomerase A3; MHC, major histocompatibility complex.

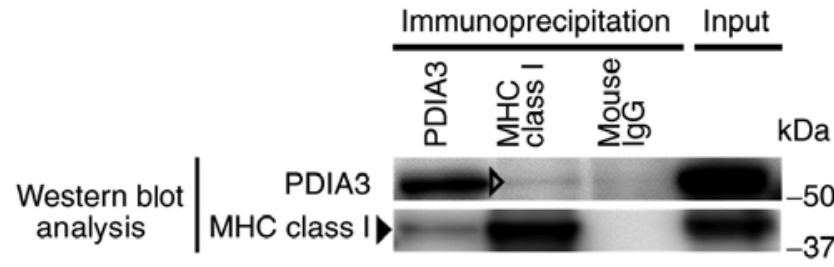

Figure 7. Co-immunoprecipitation of PDIA3 and MHC class I. In a western blot with anti-PDIA3 antibody, a faint band of co-immunoprecipitated PDIA3 was detected in the sample immunoprecipitated with anti-MHC class I antibody (open triangle). In a western blot with anti-MHC class I antibody, a faint band of co-immunoprecipitated MHC class I was detected in the sample immunoprecipitated with anti-PDIA3 antibody (closed triangle). PDIA3, protein disulfide isomerase $\mathrm{A} 3$; MHC, major histocompatibility complex.

of stage II to IV, the prognosis of PDIA3-High GC was significantly favorable compared with that of PDIA3-Low GC.

There are several differences in the study design between the present study and the previous study (11). The previous study included cases from several different ethnic groups. Furthermore, the histological and immunohistochemical evaluations were conducted using tissue microarray. However, the present study included only Japanese cases of GC. In addition, histological examinations were performed with tissue sections, which contain the whole area of carcinoma. As GC is heterogenous in histology and molecular alterations (18), it is necessary to evaluate the whole area of carcinoma. These factors may have led to the slight difference reported in the prognosis of GC. To the best of our knowledge, this is the first report on a favorable prognosis in Japanese cases of GC with highly expressed PDIA3.

There have been several reports on the association of PDIA3 expression with prognosis in types of cancer other than GC. High PDIA3 expression has been associated with a worse prognosis in laryngeal and hepatocellular carcinoma, and diffuse glioma (7-9). However, high expression has also been associated with a favorable prognosis in uterine cervical cancer (10) and GC (11). The present study also suggested the association of PDIA3 expression with favorable prognosis in GC. The biological significance of PDIA3 on the prognosis of carcinomas varies largely among carcinomas of various sites. This may reflect the difference in carcinogenesis and histology of the carcinomas. As PDIA3 exerts various cellular functions (4), the prognosis is influenced by the cellular function, in which PDIA3 serves a critical role in carcinoma cells. Cell proliferation and tumor immunity are possible cellular functions with which PDIA3 is involved.

PDIA 3 is involved in cell proliferation and stabilizing receptors on the cell membrane $(19,20)$ and intracellular signaling molecules, including signal transducer and activator of transcription 3 (21) and mechanistic target of rapamycin (22). In hepatocellular carcinoma, high expression of PDIA3 was associated with high proliferative activity and low apoptotic cell death, whereas low expression was associated low proliferative activity and high apoptotic cell death $(8,9)$. The unfavorable prognosis in hepatocellular carcinoma with elevated expression of PDIA3 may be, in part, accounted for by increased cell proliferation, which is supported by PDIA3 expression. However, in GC, there was no significant difference in the proliferation and apoptotic cell death between PDIA3-High and PDIA3-Low GC in the present study. It was thus considered that the role of PDIA3 on the proliferation or apoptotic cell death was only limited in GC. This may also reflect the difference in carcinogenic machinery between hepatocellular carcinoma (23) and GC (24). The findings suggest that the favorable prognosis in PDIA3-High GC is influenced by a factor other than cell proliferation.

PDIA3 is involved in the processing and transport of antigens to the cell membrane during an immune response $(25,26)$. Thus, the association of PDIA 3 with MHC class I was examined in culture cell lines of GC in the present study. The expression of MHC class I was indicated in three of four assessed cell lines, and the expression of MHC class I was upregulated under the stimulation of IFN $\gamma$ (17). The expression levels of PDIA3 varied largely among the cell lines, and it appeared to be comparable with the expression level in human cases of GC. Upregulation of PDIA3 was evident in one cell line, KATO-III, which had the most abundant expression of PDIA3 among the cell lines. Co-immunoprecipitation experiments demonstrated that MHC class I formed a complex with PDIA3. It is therefore conceivable that PDIA3 serves a critical role in tumor immunity, forming a complex with MHC class I in GC.

The expression of MHC class I in carcinoma cells may induce cytotoxic effects of lymphocytes and other immune cells $(27,28)$. Carcinoma cells that do not express MHC class I may evade from the cytotoxic effects of immune cells. Notably, the expression and upregulation of MHC class I was indicated in the cell line, KATO-III, that revealed high expression of PDIA3 in the present study. The expression of PDIA3 in carcinoma cells may suggest a sufficient capacity to form a complex with MHC class I and to transport antigens to the cell membrane. It may thus be speculated that a favorable prognosis of PDIA3-High GC is attributed, in part, to a sufficient immune response mediated by PDIA3. The present findings suggest that PDIA3 may serve an important role in the pathobiology of GC. The expression of PDIA3 may be a useful biomarker for the prediction of prognosis of GC.

\section{Acknowledgements}

The authors would like to acknowledge the excellent assistance of Ms. Kiyoko Kawahara, Mr. Takenori Fujii, 
Mr. Kiyoshi Teduka, Ms. Yoko Kawamoto and Ms. Taeko Kitamura of the Department of Integrated Diagnostic Pathology, Nippon Medical School (Tokyo, Japan).

\section{Funding}

No funding was received.

\section{Availability of data and materials}

All data generated or analyzed during this study are included in this published article.

\section{Authors' contributions}

TS, RW and ZN designed the study and wrote the manuscript draft. TS, RW, SK, RO performed histological examinations. TS, KI, MK conducted biochemical examinations, data analyses and statistical analyses. TS prepared the figures/tables. IF, EU and HY provided clinical data of the patients and assisted with revising the manuscript. ZN supervised the experimental design and manuscript writing, revised the manuscript, and gave the final approval of the version to be published. All authors read and approved the final manuscript.

\section{Ethics approval and consent to participate}

The study was approved by the Ethics Committee of the Nippon Medical School Hospital (Tokyo, Japan; no. 29-06-764). Informed consent was obtained from all patients.

\section{Patient consent for publication}

Informed consent was obtained from all patients.

\section{Competing interests}

The authors declare that they have no competing interests.

\section{References}

1. Hori M, Matsuda T, Shibata A, Katanoda K, Sobue T and Nishimoto H; Japan Cancer Surveillance Research Group: Cancer incidence and incidence rates in Japan in 2009: A study of 32 population-based cancer registries for the Monitoring of Cancer Incidence in Japan (MCIJ) project. Jpn J Clin Oncol 45 884-891, 2015.

2. Kim HJ and Oh SC: Novel systemic therapies for advanced gastric cancer. J Gastric Cancer 18: 1-19, 2018.

3. Song Z, Wu Y, Yang J, Yang D and Fang X: Progress in the treatment of advanced gastric cancer. Tumour Biol 39 1010428317714626, 2017.

4. Turano C, Gaucci E, Grillo C and Chichiarelli S: ERp57/GRP58: A protein with multiple functions. Cell Mol Biol Lett 16: 539-563, 2011.

5. Ni M and Lee AS: ER chaperones in mammalian development and human diseases. FEBS Lett 581: 3641-3651, 2007.

6. Hettinghouse A, Liu R and Liu CJ: Multifunctional molecule ERp57: From cancer to neurodegenerative diseases. Pharmacol Ther 181: 34-48, 2018.

7. Choe MH, Min JW, Jeon HB, Cho DH, Oh JS, Lee HG, Hwang SG, An S, Han YH and Kim JS: ERp57 modulates STAT3 activity in radioresistant laryngeal cancer cells and serves as a prognostic marker for laryngeal cancer. Oncotarget 6: 2654-2666, 2015.
8. Takata H, Kudo M, Yamamoto T, Ueda J, Ishino K, Peng WX, Wada R, Taniai N, Yoshida H, Uchida E, et al: Increased expression of PDIA3 and its association with cancer cell proliferation and poor prognosis in hepatocellular carcinoma. Oncol Lett 12: 4896-4904, 2016.

9. Zou H, Wen C, Peng Z, Shao YY, Hu L, Li S, Li C and Zhou HH: $\mathrm{P} 4 \mathrm{HB}$ and PDIA3 are associated with tumor progression and therapeutic outcome of diffuse gliomas. Oncol Rep 39: 501-510, 2018.

10. Chung H, Cho H, Perry C, Song J, Ylaya K, Lee H and Kim JH: Downregulation of ERp57 expression is associated with poor prognosis in early-stage cervical cancer. Biomarkers 18: 573-579, 2013.

11. Leys CM, Nomura S, LaFleur BJ, Ferrone S, Kaminishi M, Montgomery E and Goldenring JR: Expression and prognostic significance of prothymosin-alpha and ERp57 in human gastric cancer. Surgery 141: 41-50, 2007.

12. Lauren P: The two histological main types of gastric carcinoma: Diffuse and so-called intestinal-type carcinoma. An attempt at a histo-clinical classification. Acta Pathol Microbiol Scand 64: 31-49, 1965.

13. Washington $\mathrm{K}$ : 7th edition of the AJCC cancer staging manual: Stomach. Ann Surg Oncol 17: 3077-3079, 2010.

14. Sano T, Coit DG, Kim HH, Roviello F, Kassab P, Wittekind C, Yamamoto Y and Ohashi Y: Proposal of a new stage grouping of gastric cancer for TNM classification: International gastric cancer association staging project. Gastric Cancer 20: 217-225, 2017.

15. Pirker R, Pereira JR, von Pawel J, Krzakowski M, Ramlau R, Park K, de Marinis F, Eberhardt WE, Paz-Ares L, Störkel S, et al: EGFR expression as a predictor of survival for first-line chemotherapy plus cetuximab in patients with advanced non-small-cell lung cancer: Analysis of data from the phase 3 FLEX study. Lancet Oncol 13: 33-42, 2012.

16. Livak KJ and Schmittgen TD: Analysis of relative gene expression data using real-time quantitative PCR and the $2^{-\Delta \Delta C_{\mathrm{T}}}$ method. Methods 25: 402-408, 2001.

17. Zhou F: Molecular mechanisms of IFN-gamma to up-regulate MHC class I antigen processing and presentation. Int Rev Immunol 28: 239-260, 2009.

18. Wong SS, Kim KM, Ting JC, Yu K, Fu J, Liu S, Cristescu R, Nebozhyn M, Gong L, Yue YG, et al: Genomic landscape and genetic heterogeneity in gastric adenocarcinoma revealed by whole-genome sequencing. Nat Commun 5: 5477, 2014.

19. Doroudi M, Olivares-Navarrete R, Boyan BD and Schwartz Z: A review of $1 \alpha, 25(\mathrm{OH})_{2} \mathrm{D}_{3}$ dependent Pdia3 receptor complex components in Wnt5a non-canonical pathway signaling. J Steroid Biochem Mol Biol 152: 84-88, 2015.

20. Gaucci E, Altieri F, Turano C and Chichiarelli S: The protein ERp57 contributes to EGF receptor signaling and internalization in MDA-MB-468 breast cancer cells. J Cell Biochem 114: 2461-2470, 2013.

21. Coe H, Jung J, Groenendyk J, Prins D and Michalak M: ERp57 modulates STAT3 signaling from the lumen of the endoplasmic reticulum. J Biol Chem 285: 6725-6738, 2010.

22. Ramirez-Rangel I, Bracho-Valdés I, Vázquez-Macías A, Carretero-Ortega J, Reyes-Cruz G and Vázquez-Prado J: Regulation of mTORC1 complex assembly and signaling by GRp58/ERp57. Mol Cell Biol 31: 1657-1671, 2011.

23. Li CW, Chang PY and Chen BS: Investigating the mechanism of hepatocellular carcinoma progression by constructing genetic and epigenetic networks using NGS data identification and big database mining method. Oncotarget 7: 79453-79473, 2016.

24. Katona BW and Rustgi AK: Gastric cancer genomics: Advances and future directions. Cell Mol Gastroenterol Hepatol 3: 211-217, 2017.

25. Blees A, Januliene D, Hofmann T, Koller N, Schmidt C, Trowitzsch S, Moeller A and Tampé R: Structure of the human MHC-I peptide-loading complex. Nature 551: 525-528, 2017.

26. Garbi N, Hämmerling G and Tanaka S: Interaction of ERp57 and tapasin in the generation of MHC class I-peptide complexes. Curr Opin Immunol 19: 99-105, 2007.

27. Chen Y, Lin J, Guo ZQ, Lin WS, Zhou ZF, Huang CZ, Chen Q and Ye YB: MHC I-related chain a expression in gastric carcinoma and the efficacy of immunotherapy with cytokine-induced killer cells. Am J Cancer Res 5: 3221-3230, 2015.

28. Ribeiro CH, Kramm K, Gálvez-Jirón F, Pola V, Bustamante M, Contreras HR, Sabag A, Garrido-Tapia M, Hernández CJ, Zúñiga $\mathrm{R}$, et al: Clinical significance of tumor expression of major histocompatibility complex class I-related chains A and B (MICA/B) in gastric cancer patients. Oncol Rep 35: 1309-1317, 2016. 